\title{
Moderate Loss of the Extracellular Matrix Proteoglycan Lumican Attenuates Cardiac Fibrosis in Mice Subjected to Pressure Overload
}

\author{
Naiyereh Mohammadzadeh ${ }^{a, b} \quad$ Arne Olav Melleby $^{a, b} \quad$ Sheryl Palmero ${ }^{a, b}$ \\ Ivar Sjaastad ${ }^{a, b}$ Shukti Chakravarti ${ }^{c, d}$ Kristin V.T. Engebretsen ${ }^{e}$ \\ Geir Christensen $^{a, b}$ Ida G. Lunde ${ }^{a, b, f}$ Theis Tønnessen ${ }^{a, b, g}$ \\ anstitute for Experimental Medical Research, Oslo University Hospital and University of Oslo, Oslo, Norway; \\ ${ }^{b}$ KG Jebsen Center for Cardiac Research and Center for Heart Failure Research, University of Oslo, Oslo, Norway; \\ 'Department of Medicine, Johns Hopkins University, Baltimore, MD, USA; ${ }^{\mathrm{d}}$ Department of Ophthalmology and \\ Pathology, NYU Langone Health, New York, NY, USA; ${ }^{\circ}$ Department of Surgery, Vestre Viken Hospital, Drammen, \\ Norway; ${ }^{\mathrm{f} C e n t e r}$ for Molecular Medicine Norway, Oslo University Hospital and University of Oslo, Oslo, Norway; \\ ${ }^{9}$ Department of Cardiothoracic Surgery, Oslo University Hospital, Oslo, Norway
}

\section{Keywords}

Lumican · Aortic banding $\cdot$ Cardiac remodeling $\cdot$ Cardiac fibrosis

\begin{abstract}
Introduction: The heart undergoes myocardial remodeling during progression to heart failure following pressure overload. Myocardial remodeling is associated with structural and functional changes in cardiac myocytes, fibroblasts, and the extracellular matrix (ECM) and is accompanied by inflammation. Cardiac fibrosis, the accumulation of ECM molecules including collagens and collagen cross-linking, contributes both to impaired systolic and diastolic function. Insufficient mechanistic insight into what regulates cardiac fibrosis during pathological conditions has hampered therapeutic solutions. Lumican (LUM) is an ECM-secreted proteoglycan known to regulate collagen fibrillogenesis. Its expression in the heart is increased in clinical and experimental heart fail-
\end{abstract}

ure. Furthermore, LUM is important for survival and cardiac remodeling following pressure overload. We have recently reported that total lack of LUM increased mortality and left ventricular dilatation, and reduced collagen expression and cross-linking in LUM knockout mice after aortic banding $(A B)$. Here, we examined the effect of LUM on myocardial remodeling and function following pressure overload in a less extreme mouse model, where cardiac LUM level was reduced to $50 \%$ (i.e., moderate loss of LUM). Methods and $\boldsymbol{R e}$ sults: mRNA and protein levels of LUM were reduced to $50 \%$ in heterozygous LUM (LUM+/-) hearts compared to wildtype (WT) controls. LUM+/- mice were subjected to $A B$. There was no difference in survival between LUM+/- and WT mice post-AB. Echocardiography revealed no striking differences in cardiac geometry between LUM+/- and WT mice 2, 4 , and 6 weeks post-AB, although markers of diastolic dysfunction indicated better function in LUM+/- mice. LUM+/hearts revealed reduced cardiac fibrosis assessed by histology. In accordance, the expression of collagen I and III, the

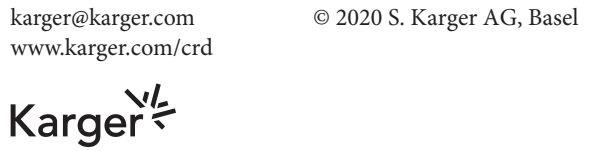

Prof. Theis Tønnessen

Institute for Experimental Medical Research and Department of Cardiothoracic Surgery Oslo University Hospital, Kirkeveien 166 NO-0407 Oslo (Norway)

E-Mail theis.tonnessen@medisin.uio.no 
main fibrillar collagens in the heart, and other ECM molecules central to fibrosis, i.e. including periostin and fibronectin, was reduced in the hearts of LUM+/- compared to WT 6 weeks post- $A B$. We found no differences in collagen crosslinking between $L U M+/-$ and WT mice post- $A B$, as assessed by histology and qPCR. Conclusions: Moderate lack of LUM attenuated cardiac fibrosis and improved diastolic dysfunction following pressure overload in mice, adding to the growing body of evidence suggesting that LUM is a central profibrotic molecule in the heart that could serve as a potential therapeutic target.

(c) 2020 S. Karger AG, Basel

\section{Introduction}

Left ventricular pressure overload leads to increased wall stress, cardiac remodeling, and ultimately heart failure if left untreated. Cardiac remodeling encompasses molecular, cellular, and interstitial changes that manifest clinically as alterations in geometry and function of the heart following cardiac load or injury. Cardiac remodeling includes alterations at the level of cardiac myocytes, cardiac fibroblasts (CFB), and the extracellular matrix (ECM) [1]. Primarily, structural modifications of the heart exert a compensatory effect to maintain normal cardiac function [2,3]; however, sustained stress and remodeling lead to progressive and irreversible dysfunction and failure of the heart [4].

During cardiac remodeling in response to pressure overload, cardiac fibrosis is induced, affecting the structure and function of the heart $[5,6]$. Excessive accumulation of fibrillar collagens and collagen cross-linking lead to cardiac fibrosis characterized by wall stiffening and impaired systolic and diastolic function [7]. There is insufficient mechanistic insight into what regulates cardiac fibrosis. Thus, development of effective drugs to treat cardiac fibrosis and improve cardiac function is hampered.

Proteoglycans are proteins substituted with covalently attached glycosaminoglycan chains and are major components of the ECM along with collagens [8]. Proteoglycans such as syndecans, glypicans, fibromodulin (FMOD), and biglycan play important roles in pressure overloaddriven cardiac remodeling, including hypertrophic growth, fibrosis, and inflammation [9-13]. Lumican (LUM) is a keratan sulfate small leucine-rich proteoglycan localized to the ECM. Like FMOD, it binds directly to fibrillar collagens [14] and regulates collagen fibrillogenesis in tissues such as the cornea, tendon, and skin [15].
LUM levels are increased in fibrotic lesions of patients with pulmonary fibrosis and human atherosclerotic coronary arteries [16, 17], and LUM is important for progression of hepatic fibrosis [18]. It is expressed in the developing heart $[19,20]$, and we have previously shown that cardiac LUM levels are increased in clinical and experimental heart failure [21-23]. Recently, we have shown that LUM knockout mice (LUMKO) exhibit increased early mortality, exacerbated left ventricular dilatation, and reduced contractility following pressure overload [24]. Interestingly, collagen cross-linking and cardiac collagen expression are reduced in LUMKO mice following pressure overload, suggesting a role in cardiac fibrosis [24]. In line with this, it has been suggested that attenuated expression of the proteoglycans LUM, FMOD, and decorin in CXCR5-deficient mice leads to LV dilatation and heart failure following pressure overload [22]. Thus, these data suggest that LUM is essential for preserving myocardial function and ECM structure in pressure overload.

Here, we studied the effect of moderately reduced LUM levels in the heart. LUMKO mice are extremely fragile and show high perinatal death $[20,24]$. Since total lack of LUM is nonphysiological, has developmental effects, is lethal in itself in most of the embryos, and the surviving mice show increased mortality upon myocardial stress, interpretation of cardiac function from LUM$\mathrm{KO}$ data remains difficult along with low numbers of available animals. Previous studies have shown that LUM expression levels are regulated by gene dosage effects, with heterozygous LUM+/- mice having 50\% of LUM levels seen in wild-type (WT) mice [25]. Thus, in the present study, we subjected heterozygous LUM+/- mice to pressure overload in vivo to examine how moderate reduction in cardiac LUM levels affects survival, cardiac remodeling, and function.

\section{Methods}

\section{Animal Experiments}

Animal experiments were approved by The Norwegian Animal Research Committee (protocol IDs 4531 and 11669), and conformed to the Guide for the Care and Use of Laboratory Animals (National Institute of Health (NIH, MD)). Heterozygous $\mathrm{LUM}+/-$ and WT littermates were produced from heterozygous intercrosses of LUM+/- mice, and genotyping was performed on ear biopsies at weaning age (3-4 weeks [w] of age) [24]. Adult (9-10 w old) male WT and LUM+/- mice were subjected to banding of the ascending aorta $(\mathrm{AB})$ by inserted $\mathrm{O}$-ring with inner diameter of $0.61 \mathrm{~mm}(\mathrm{AB})$ and a nitrile rubber piece (SHAM), as previously described [26]. Mice were intubated and ventilated 


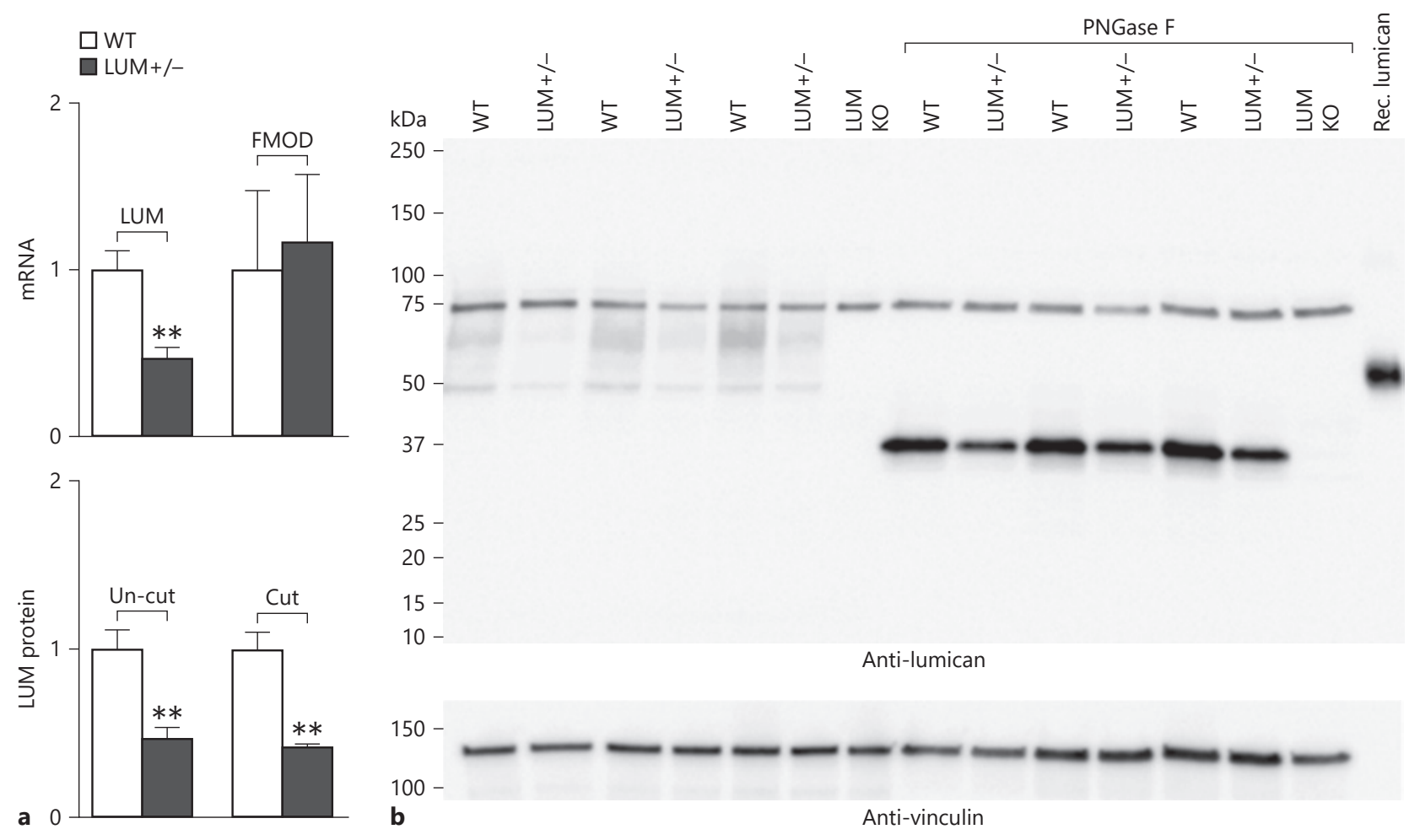

Fig. 1. Lumican (LUM) expression was reduced in hearts of LUM+/- mice. Relative mRNA levels of LUM and FMOD $(n=$ $5-7 ; \mathbf{a})$, and protein levels of LUM $(n=7 ; \mathbf{b})$ in the left ventricle of untreated, adult heterozygous LUM+/-, and WT control mice without (uncut) and with (cut) deglycosylation treatment with PNGase F, an enzyme which separates all the glycosaminoglycan chains from the core protein. mRNA was normalized to expression of ribosomal protein L32 (RPL32), while vinculin was used for pro-

with a mixture of $98 \%$ oxygen and $2 \%$ isoflurane on a Mini-Vent ventilator (Harvard Apparatus, Holliston, MA, USA). AB was performed through a left-sided, muscle-saving thoracotomy under a dissecting microscope (Carl Zeiss Microscopy GmbH, Jena, Germany). Mice received pre- and postoperative analgesia by subcutaneous injection of $0.02 \mathrm{~mL}(0.3 \mathrm{mg} / \mathrm{mL})$ buprenorphine. Animals with sufficient degree of aortic constriction (maximal flow velocity [Vmax] $4 \mathrm{~m} / \mathrm{s}$ over the stenosis) $24 \mathrm{~h}$ post- $\mathrm{AB}$ were included. Echocardiography was performed by an experienced researcher blinded to genotype before $\mathrm{AB}$ (baseline), and 2, 4 and 6 $\mathrm{w}$ after $\mathrm{AB}$, on mice breathing a mixture of $1.75 \%$ isoflurane and $98.25 \% \mathrm{O}_{2}$ on a mask, using the VEVO 2100 system (VisualSonics, Toronto, ON, Canada). Mice were anesthetized with $5 \%$ isoflurane and sacrificed by cervical dislocation $6 \mathrm{w}$ post-AB. Heart and lungs were rapidly excised, rinsed in PBS and blotted dry. Heart and lungs were weighed, and normalized to body weight. Hearts were cut with a razor blade at the mid-ventricular plane. The basal biventricular part was used for histology, while from the apical part, the LV was dissected, snap-frozen in liquid nitrogen and

Lumican in Cardiac Remodeling

following Pressure Overload tein loading control. For immunoblotting, recombinant (Rec.) LUM was used as positive control and left ventricles from LUMKO mice as negative control. LUM appears as a $37-\mathrm{kDa}$ core protein after de-glycosylation with PNGase F, and was quantified both as total glycosylated LUM (50- to 75-kDa smear) (uncut) and $37-\mathrm{kDa}$ core protein (cut). The $75-\mathrm{kDa}$ band is unspecific. Data are presented as mean \pm SEM. Statistical differences were tested using an unpaired $t$ test vs. WT, ${ }^{* *} p<0.01{ }^{*} p<0.05$.

stored at $-70^{\circ}$ for RNA or protein analyses. Under basal conditions, no obvious phenotypical differences were observed between $\mathrm{LUM}+/-$ and WT mice.

\section{Histology and Quantification}

Wheat germ agglutinin staining was performed to measure cardiomyocyte cross-sectional area as previously described [24]. Picrosirius Red staining was performed to measure fibrosis and collagen cross-linking using bright field and polarized light, respectively, [10] as described [24]. Image J was used for quantification as described [24].

\section{Gene Expression Analysis}

Total RNA was extracted, cDNA synthesized, and Fast Real Time PCR System or ddPCR was performed, as described [24].

\section{Protein Analysis}

Immunoblotting was performed on protein lysates from cells and LV tissues, as described $[9,11,27]$. Enzymatic deglycosylation 


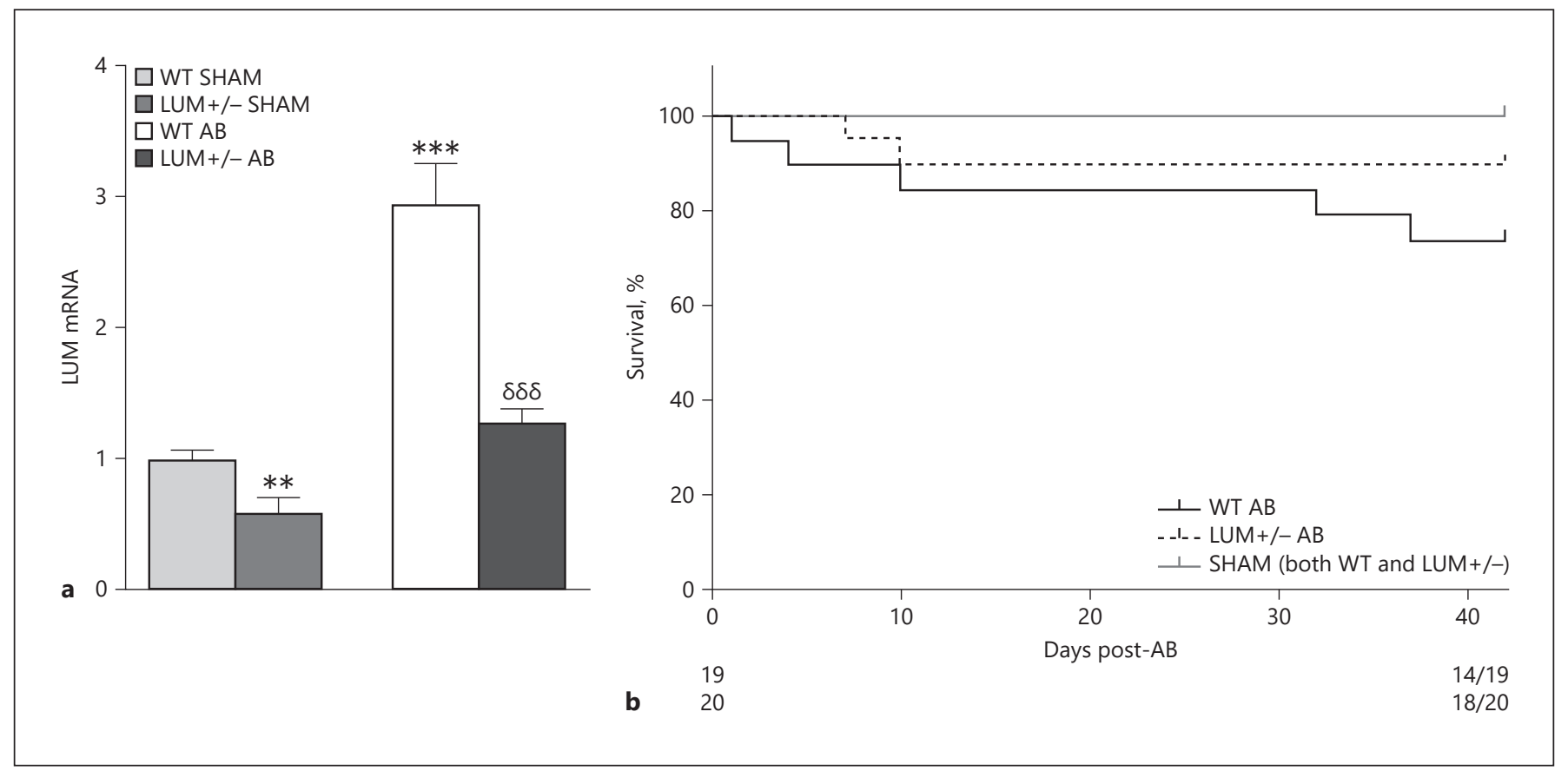

Fig. 2. No difference in survival between LUM+/- and WT following pressure overload. a Relative left ventricular mRNA levels of LUM in LUM+/- and WT mice 6 weeks (w) post-AB. mRNA expressions were normalized to expression of ribosomal protein L32 (RPL32). b Kaplan-Meier survival curve showing \% survival of heterozygous LUM+/- and WT mice up to $6 \mathrm{w}$ post-aortic band-

of LUM was performed using PNGase F $\left(1 \mu \mathrm{L} / 1 \mathrm{~h} / 37^{\circ} \mathrm{C}\right)$, as described $[24,28]$.

\section{Statistics}

Data are expressed as mean \pm standard error of the mean. Statistical analysis was performed using the GraphPad software (Prism 7). $p<0.05$ was considered as statistically significant. The use of parametric or nonparametric tests was based on results from analyses of distributions. Statistical significance was determined using Student's $t$ test and one-way ANOVA with Dunn's post hoc test and Mann-Whitney rank sum test. Survival rates were calculated using the log-rank (Mantel-Cox) test.

\section{Results}

\section{LUM Expression Was Reduced in Hearts of}

Heterozygous LUM+/- Mice

Cardiac LUM levels were assessed in the hearts of LUM+/- and WT control mice. As expected, cardiac LUM mRNA was reduced (0.46-fold) in LUM+/- versus WT (Fig. 1a). We found no compensatory change in cardiac FMOD mRNA levels (Fig. 1a), a closely related keratan sulfate proteoglycan [13, 29-31]. Glycosylated LUM ing $(\mathrm{AB})$ or SHAM operation. All SHAM-operated LUM+/- and WT mice survived. Number of surviving mice is noted under the graph, $n$ SHAM $=8-10$. Statistical differences were tested using one-way ANOVA with Dunn's post hoc test vs. WT SHAM, ${ }^{* * *} p<0.005,{ }^{* *} p<0.01$, or an unpaired $t$ test LUM+/- vs. WT AB, ${ }^{\delta \delta \delta} p<0.005$ (a), and log rank (Mantel-Cox) test vs. WT AB (b). was, as expected [21,24], detected as a $50-$ to $75-\mathrm{kDa}$ protein in protein lysates from $\mathrm{LUM}+/-$ and WT hearts (Fig. 1b), and we detected no nonglycosylated LUM in the heart tissue (37-kDa core protein) (Fig. 1b). LUM protein levels in the hearts were quantified in two ways: from untreated heart lysates (glycosylated LUM at 50-75 kDa) and after PNGase F treatment of lysates (37-kDa band). In line with the observed LUM mRNA reduction (Fig. 1a), quantification of both untreated and deglycosylated heart samples showed a comparable reduction of LUM protein in LUM+/- hearts (reduced to 0.55-fold and 0.67, respectively, vs. WT mice) (Fig. 1a, b).

\section{No Difference in Survival between LUM+/- and WT} Mice following Pressure Overload

To assess the role of LUM in heart failure progression and cardiac remodeling, heterozygous LUM knockout $(\mathrm{LUM}+/-)$ and WT adult mice were subjected to pressure overload by $\mathrm{AB}$. In accordance with our previously published data [21], left ventricular LUM levels were increased in WT mice after $A B$ compared to respective SHAM controls (Fig. 2a). Following 6 w of AB, LUM+/- 
mice showed comparable mortality compared to WT (Fig. $2 \mathrm{~b}, p=0.19$ ), indicating that a moderate reduction in cardiac LUM level does not impact significantly on survival following pressure overload within the time frame examined. There was no difference in survival between the two genotypes post-SHAM operation (Fig. 2b). Thus, comparable survival in LUM+/- and WT mice upon pressure overload suggested that studying the effects of moderate reduction in LUM levels in the heart would be possible without mortality as a confounding factor, as seen in the LUMKO mice [24].

Cardiac Function and Geometry of LUM+/- and WT Mice following Pressure Overload

Echocardiography was performed in adult, untreated LUM+/- mice and WT littermate controls, and we observed no differences in cardiac dimensions and function at baseline (Table 1). Serial echocardiography was performed at 2, 4, and $6 \mathrm{w}$ post- $\mathrm{AB}$, and hearts and lungs were harvested at $6 \mathrm{w}$ post-AB for molecular analysis. Overall, postmortem organ weights and echocardiography revealed similar cardiac phenotypes in $\mathrm{LUM}+/-$ and WT at 2,4 , or $6 \mathrm{w}$ post-AB (Table 1 ).

LUM+/- and WT mice developed hypertrophic remodeling compared to baseline WT 2 and $4 \mathrm{w}$ post-AB, and compared to SHAM WT controls $6 \mathrm{w}$ post-AB (Table 1). Hypertrophic remodeling was measured by interventricular septum and posterior wall thicknesses, and relative wall thickness. Hypertrophic remodeling was increased to a similar degree in LUM+/- and WT compared to baseline WT and SHAM controls times post-AB, as assessed by echocardiography (Table 1 ). In line with this, the cross-sectional area of cardiomyocytes, assessed by histology, and postmortem heart weight (Fig. 3a, b) were increased to a similar degree in LUM+/- and WT compared to SHAM $6 \mathrm{w}$ post-AB.

During progression of cardiac remodeling from $2-6 \mathrm{w}$ post- $A B$, left ventricular inner diameter (LVID) was not altered in LUM+/- and WT mice compared to baseline WT and SHAM controls (Table 1). Contractile function assessed as fractional shortening (FS\%) was reduced to a comparable degree in LUM+/- and WT compared to SHAM WT $6 \mathrm{w}$ post-AB (Table 1). The echocardiographic measures used to estimate myocardial compliance and diastolic function, MVE and Mdec, were significantly lower in LUM+/- mice than in WT mice $6 \mathrm{w}$ post-AB, indicating more severe diastolic dysfunction in the latter (Table 1). Lung weight (Fig. 3b) and left atrial diameter (Table 1) were similarly increased in both genotypes compared to SHAM WT at $6 \mathrm{w}$ post-AB, suggesting sim-

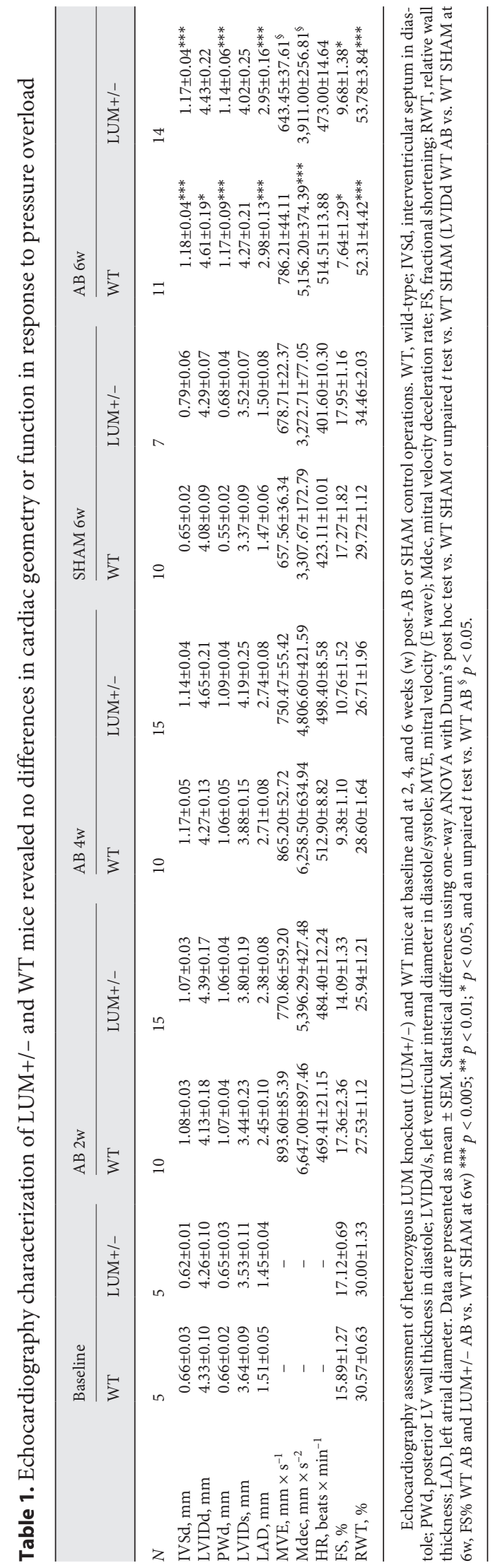

Lumican in Cardiac Remodeling

following Pressure Overload
Cardiology 2020;145:187-198 DOI: $10.1159 / 000505318$ 
ilar degree of congestion. As expected with heart failure, the cardiac expression of signature heart failure molecules ANP and BNP (encoded by NPPA and NPPB, respectively) were increased (Fig. 3c) after $\mathrm{AB}$, and in accordance with echocardiography data and postmortem organ weights, increased in both genotypes to a comparable extent. We have recently shown by RNA sequencing that the heart failure signature molecule GDF15 is among the top 10 most downregulated molecules in LUM KO
$2 \mathrm{w}$ post- $\mathrm{AB}[24]$, and in accordance with reduced LUM levels in the hearts of LUM+/- mice, mRNA levels of GDF15 were reduced versus WT $6 \mathrm{w}$ post-AB (Fig. 3c).

\section{Reduced Cardiac Fibrosis in LUM+/- Mice after Pressure Overload}

Importantly, we examined whether a moderate reduction in LUM levels altered fibrotic remodeling after pressure overload. As expected, the fibrotic area was increased

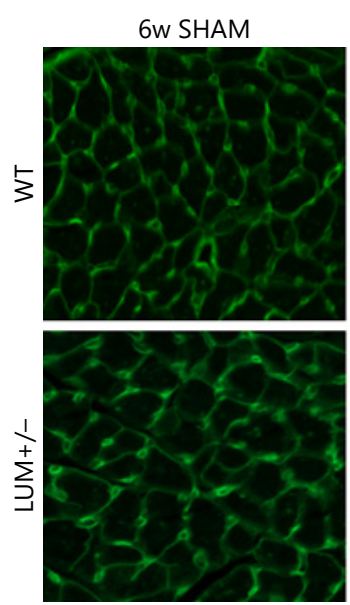

a

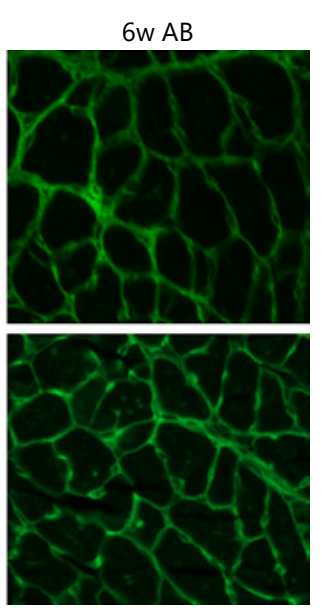

$\overline{50 \mu \mathrm{m}}$
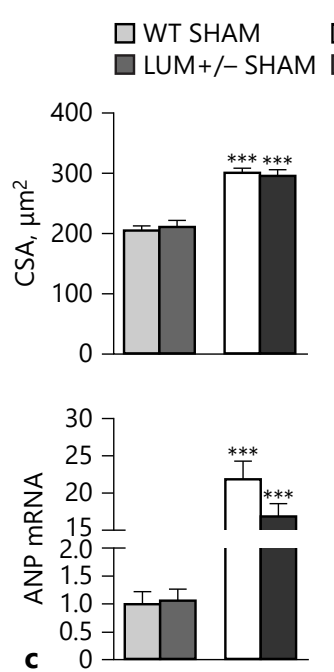

$\square$ WT AB

LUM+/- AB
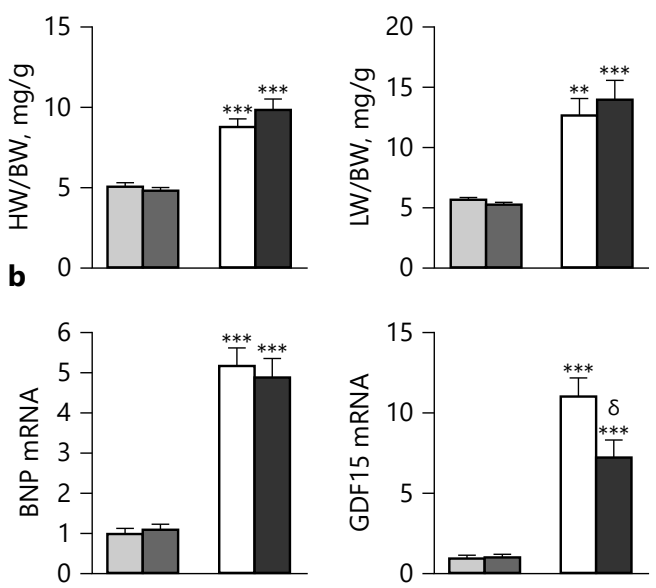

Fig. 3. Similar hypertrophic growth in LUM+/- and WT mice after pressure overload. Heterozygous lumican knockout (LUM+/-) and WT mice were subjected to aortic banding (AB) or SHAM operation, and the left ventricle harvested for histology and molecular analyses 6 weeks (w) post-surgery. a Representative images of mid-ventricular histology sections stained with wheat germ agglutinate to measure cardiomyocyte cross-sectional areas (CSA) ( $n$ SHAM $=8-10, n \mathrm{AB}=14-17)$. Scale bar $=50 \mu \mathrm{m}$. b Heart and lung weights were normalized to body weight (HW/BW and LW/BW, respectively) ( $n$ SHAM $=8-10, n \mathrm{AB}=14-17)$. c Relative left ventricular mRNA levels of signature molecules of heart failure, ANP and BNP (encoding atrial and brain natriuretic peptides, respectively), and growth differentiation factor (GDF) 15 ( $n$ SHAM = $8-10, n \mathrm{AB}=14-17)$. mRNA expression was normalized to expression of ribosomal protein L32 (RPL32). Data are presented as mean \pm SEM. Statistical differences were tested using one-way ANOVA with Dunn's post hoc test vs. WT SHAM, ${ }^{* * *} p<0.005$; ${ }^{* *} p<0.01$; or an unpaired $t$ test LUM+/- vs. WT AB, ${ }^{\delta} p<0.05$.
Fig. 4. Reduced collagen deposition in hearts of LUM+/- mice after pressure overload. Heterozygous lumican knockout (LUM+/-) and WT mice were subjected to aortic banding (AB) or SHAM operation, and the left ventricle was harvested for histology and molecular analyses 6 weeks (w) post-surgery. a Representative images of mid-ventricular histology sections stained with Picrosirius red visualizing fibrillar collagens. Fibrotic area $=$ area of red staining/total area in \% $(n$ SHAM $=8-9, n \mathrm{AB}=14-15)$. Scale bar 50 $\mu \mathrm{m}$. b-f, h Relative left ventricular (LV) mRNA levels of fibrillar collagens I and III (COL1A2 and COL3A1, respectively), extracellular matrix protein periostin (POSTN) and collagen-binding protein fibronectin (FN), transforming growth factor beta (TGF $\beta$ ), collagen cross-linking enzyme lysyl oxidase LOX, matrix metallo- proteinases-9 (MMP9) and -2 (MMP2), myofibroblast differentiation markers $\alpha$-smooth muscle actin ( $\alpha$ SMA, encoded by ACTA2) and SM22, proliferating cell nuclear antigen (PCNA), and proteoglycans fibromodulin (FMOD), decorin (DCN), and biglycan (BGN) $(n$ SHAM $=8-10, n \mathrm{AB}=14-17)$. g Representative immunoblots and quantitative total levels of SMAD2/3 and NFKB inhibitor IKBa in the LV of LUM+/- and WT $6 \mathrm{w}$ post-AB. mRNA expression was normalized to expression of ribosomal protein L32 (RPL32). Coomassie staining was used as a loading control. Data are presented as mean \pm SEM. Statistical differences were tested using one-way ANOVA with Dunn's post hoc test vs. WT SHAM, ${ }^{* * *} p<0.005$; ${ }^{* *} p<0.01 ;{ }^{*} p<0.05$, or an unpaired $t$ test vs. WT $\mathrm{AB},{ }^{\delta \delta} p<0.01 ;{ }^{\delta} p<0.05$.

(For figure see next page.) 


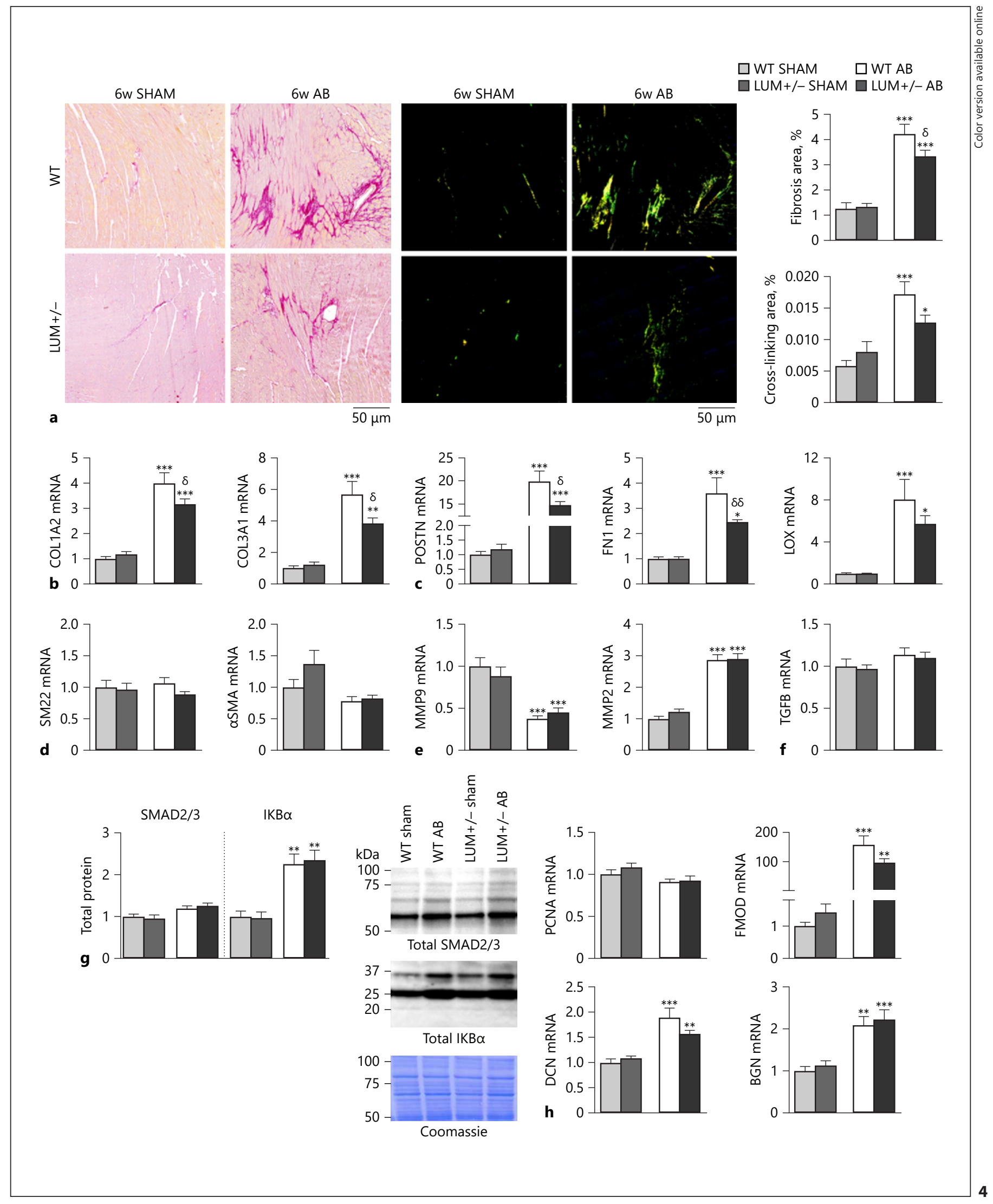




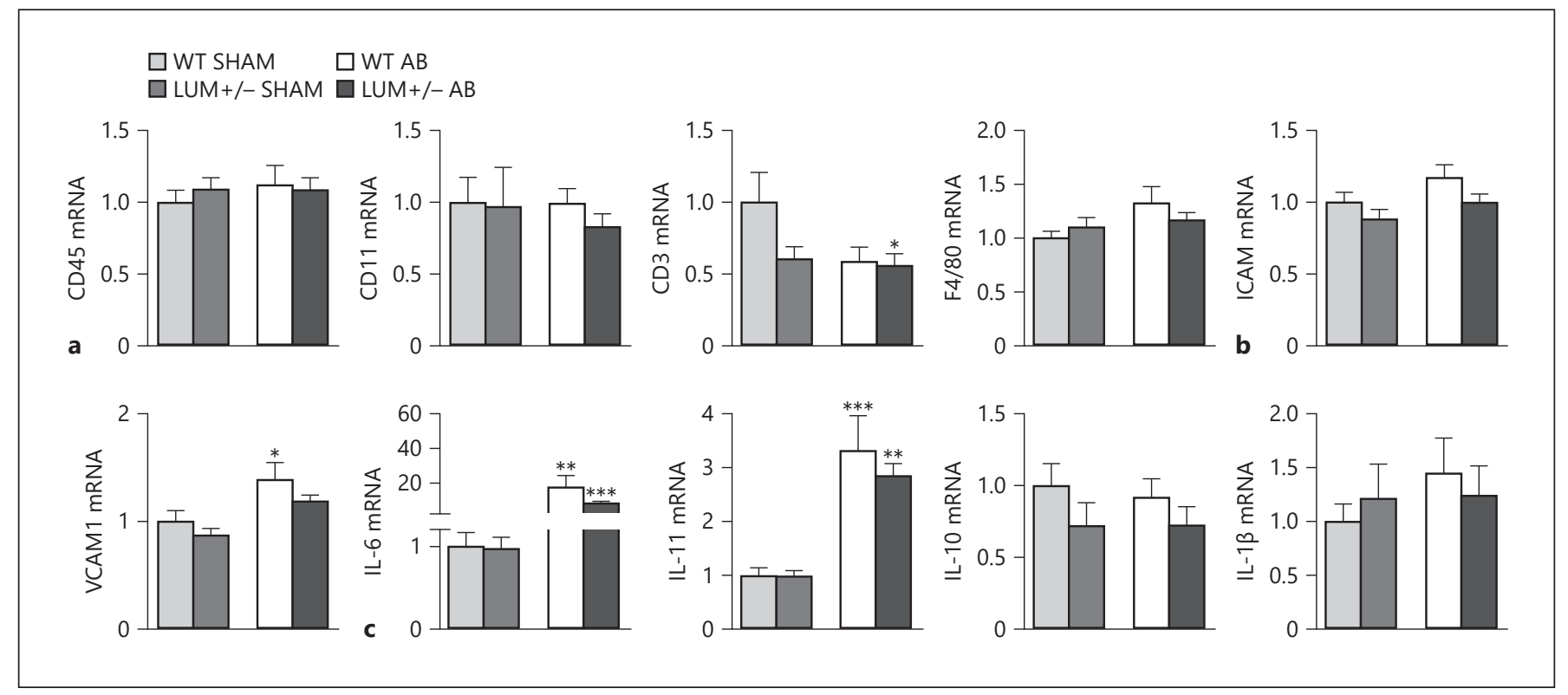

Fig. 5. No exacerbation of immune cell infiltration in $\mathrm{LUM}+/-$ mice post-AB. a-c Left ventricular mRNA expression of immune cell surface markers: leukocytes, CD45 (encoding cluster of differentiation 45) and CD11a (encoding cluster of differentiation 11), T-cells, CD3 (encoding cluster of differentiation 3), and macrophages, F4/80 (encoding adhesion G protein-coupled receptor E1) and immune cell adhesion molecules (ICAM1 and VCAM1, en- coding intercellular adhesion molecule-1 and vascular cell adhesion molecule 1, respectively), and interleukin-6 (IL-6), -11(IL11 ), -10 (IL-10), and -1 $\beta$ (IL-1 $\beta$ ) in LUM+/- and WT $6 \mathrm{w}$ post-AB. Statistical differences were tested using one-way ANOVA with Dunn's post hoc test vs. WT SHAM, ${ }^{* * *} p<0.005$; $^{* *} p<0.01$; $* p<0.05$.

groups (Fig. 4d). mRNA levels of the central profibrotic transforming growth factor beta (TGF $\beta$ ), and its downstream transcription factor levels of SMAD2/3 were not altered between LUM+/- and WT post-AB (Fig. 4f, g). Finally, we found no difference in the activation level of the proinflammatory transcription factor NFkB (Fig. 4g). Expression levels of matrix metalloproteinase-9 (MMP9) and -2 (MMP2), proteinases involved in fibrosis, were not different between LUM+/- and WT (Fig. 4e). Expression of the proliferating cell nuclear antigen PCNA was unaltered in $\mathrm{LUM}+/$ - and WT post-AB versus SHAM, and we found no differences between the two genotypes (Fig. 4h), suggesting no difference in cell proliferation. Finally, the expression of the ECM proteoglycans FMOD, decorin, and biglycan were similarly increased in the hearts of LUM+/- and WT $6 \mathrm{w}$ post-AB (Fig. 4h).

\section{Inflammation in LUM+/- Mice after Pressure \\ Overload}

Finally, we examined whether a moderate reduction in LUM levels altered inflammation in LUM+/- and WT hearts $6 \mathrm{w}$ post- $\mathrm{AB}$ or SHAM. We found that mRNA expression of immune cell surface markers leukocytes, 
CD45 (encoding cluster of differentiation 45) and CD11a (encoding cluster of differentiation 11), T cells, CD3 (encoding cluster of differentiation 3 ), and macrophages F4/80 (encoding adhesion G protein-coupled receptor E1) were unaltered by $A B 6 \mathrm{w}$ versus SHAM controls (Fig. 5a) with no differences between the two genotypes except for a moderate reduction in $\mathrm{CD} 3$ level in $\mathrm{AB} 6 \mathrm{w}$ $\mathrm{LUM}+/$ - versus SHAM controls (Fig. $5 \mathrm{a}$ ). We found no difference in the expression of immune cell adhesion molecules (ICAM1 and VCAM1, encoding intercellular adhesion molecule- 1 and vascular cell adhesion molecule 1 , respectively) between $\mathrm{LUM}+/-$ and $\mathrm{WT} 6 \mathrm{w}$ post- $\mathrm{AB}$ (Fig. 5b). The mRNA level of ICAM was unaltered in both genotypes by $\mathrm{AB} 6 \mathrm{w}$ versus SHAM controls; however, VCAM was increased in WT versus SHAM controls at this time point (Fig. 5b). Moreover, expression of interleukin (IL)-6 and IL-11 was similarly increased in the hearts of LUM+/- and WT $6 \mathrm{w}$ post-AB versus SHAM controls, with no differences between the two genotypes (Fig. 5 c). The mRNA levels of IL-1 $\beta$ and IL-10 were unaltered $6 \mathrm{w}$ post-AB versus SHAM controls and between the two genotypes (Fig. $5 \mathrm{c}$ ). These data indicate that a moderate reduction in LUM level does not affect inflammation after pressure overload in vivo.

\section{Discussion}

In the present study, we investigated whether moderate loss of LUM plays a role in survival and cardiac remodeling and function following pressure overload. LUM+/- mice, with approximately 50\% reduced LUM levels in the heart, were subjected to pressure overload by AB. LUM+/- and WT control mice showed comparable mortality upon pressure overload, with comparable hypertrophic remodeling and failure. Echocardiography indicated less diastolic dysfunction in LUM+/- compared to WT mice $6 \mathrm{w}$ post-AB. Importantly, cardiac fibrosis, i.e. collagen mRNA levels and protein deposition, were reduced in $\mathrm{LUM}+/-$ compared to WT hearts post- $\mathrm{AB}$, along with reduced mRNA expression of other matrix components such as POSTN and FN. Thus, the current study of LUM+/- mice adds to the growing body of evidence indicating that the ECM proteoglycan LUM regulates cardiac fibrosis, and that there is a gene dosage effect of LUM with regard to its effects on survival and cardiac remodeling after pressure overload.

We and others have previously shown that LUMKO mice, with a total lack of LUM, exhibit substantial perinatal mortality [20, 24]. Approximately 50\% of LUMKO

Lumican in Cardiac Remodeling

following Pressure Overload mice die within $24 \mathrm{~h}$ after birth [20]. Furthermore, surviving LUMKO mice show a very fragile phenotype including corneal opacity, skin and tendon fragility, and low body weight $[15,32,33]$. When subjected to stress such as pressure overload, LUMKO mice also show increased early mortality with $24 \%$ and $62 \%$ mortality at $24 \mathrm{~h}$ and $2 \mathrm{w}$ post- $\mathrm{AB}$, respectively, compared to 12 and $36 \%$ of WT controls [24]. This results in low numbers of viable mice when using LUMKO mice, which is time- and cost-consuming and might result in selection bias. To circumvent this obstacle when studying the role of LUM in cardiac remodeling in vivo, we have here used heterozygous LUM+/- mice with approximately 50\% reduction in LUM expression. To the best of our knowledge, no studies have reported the effect of moderately reduced LUM on cardiac remodeling.

LUM is important for survival, pressure overload-induced survival, and heart function as shown in our previous study through studying LUMKO mice [24]. Here, we found no significant difference in survival rate between $\mathrm{LUM}+/-$ and WT before or after pressure overload in the time frame examined in this study, although a type 2 statistical error cannot be completely excluded $(p=0.19)$. We found that adult LUM+/- mice have a comparable cardiac phenotype to WT under basal conditions. Upon pressure overload, LUM+/- and WT mice showed similarly increased heart weight and cardiomyocyte size compared to SHAM, indicating similar degree of hypertrophic remodeling. Moreover, cardiac contractile function, assessed as fractional shortening (FS\%), and lung weights were altered to the same extent in LUM+/- and WT mice compared to SHAM WT. Increased lung weights and reduced contractile function were consistent with congestive heart failure in both genotypes compared to SHAM [34]. Mdec and MVE were lower in LUM+/- mice compared to WT $6 \mathrm{w}$ post-AB indicating a stiffer myocardium and more pronounced diastolic dysfunction in the latter group. There was no difference in dilatation and FS\% between LUM+/- and WT mice post-AB. In contrast, LUMKO mice exhibit increased dilatation, hypertrophic remodeling, and exacerbated contractile dysfunction compared to WT mice post-AB [24]. These data indicate that whereas total LUM deficiency alters cardiac geometry and function, this is not the case with moderately reduced levels, suggesting a gene dosage effect.

An important finding in our study was that cardiac fibrosis is reduced in LUM+/- compared to WT hearts after pressure overload. We showed that mRNA expression and protein accumulation of the major cardiac collagens were reduced in the hearts of LUM+/- compared to WT 
post-AB. Supporting a direct role for LUM in cardiac fibrosis, we have previously shown that overexpression of LUM in CFB in vitro induces the expression of these collagens [24]. The importance of LUM in fibrosis has also been established in other tissues. LUM tissue levels are increased in fibrotic lesions of experimental and clinical models of cardiac, liver, and pulmonary fibrosis $[16,18$, 21,22 ]. Krishman et al. [18] have shown that lack of LUM attenuates hepatic fibrosis in mice subjected to hepatic injury probably due to impaired collagen fibrillogenesis. Moreover, LUM is known to induce fibrocyte differentiation, hence promote fibrosis in vitro [16]. Together with increased collagen production, increased collagen crosslinking is known to strengthen the cardiac matrix, leading to stiffening of the heart and hence cardiac dysfunction $[35,36]$. Concordantly, we have shown that LUM directly upregulates the expression of the collagen cross-linking enzyme lysyl oxidase in CFB in vitro, and LUMKO hearts exhibit impaired collagen cross-linking compared to WT following pressure overload. We therefore hypothesized that moderate lack of LUM would affect cross-linking, but we were unable to demonstrate this at the level of statistical significance. Thus, moderate reduction in LUM affects the levels of collagens more than cross-linking.

Similar to LUM, POSTN and FN expressions have been shown to increase in several heart diseases [37-41]. POSTN is known to be involved in collagen fibrillogenesis and cardiac valve development $[40,41]$, and FN is known to have a key role in progression of liver and lung fibrosis [42-44]. We have exhibited that LUM directly upregulates the expression of POSTN and FN in CFB in vitro [24]. Here, we show decreased expression of POSTN and FN in the hearts of LUM+/- compared to WT mice. Suppression of POSTN and FN reduces cardiac fibrosis in murine models of heart failure, and POSTN has been suggested as a biomarker of ECM remodeling and myocardial fibrosis $[40,45,46]$. Together, these data indicate that LUM deficiency reduces cardiac fibrosis, at least partly, through regulating POSTN and FN. POSTN and FN are known to regulate and affect the TGF $\beta / S M A D$ signaling pathway in different diseases [42-44, 47-49]. POSTN deficiency has been shown to attenuate hepatic fibrosis through regulating TGF $\beta$ signaling [49]. Although activation of TGF $\beta / S M A D$ signaling pathway is a key contributor to cardiac fibrosis following pressure overload [50], we were unable to demonstrate any effect of moderately reduced LUM on TGF $\beta, S M A D$, and CFB activation in the present study. Thus, we speculate that the reduced cardiac fibrosis in $\mathrm{LUM}+/-$ mice post- $\mathrm{AB}$ might at least partly be independent on the TGF $\beta / S M A D$ pathway, or that the regulation occurs before the examined time point at $6 \mathrm{w}$ after $\mathrm{AB}$.

MMP2 and -9 are regulated during fibrosis and inflammation, and reduced activity of MMP2 has been shown to attenuate cardiac fibrosis in experimental models of diabetic cardiomyopathy [51-53]. LUM directly upregulates the expression of MMP2 in CFBs in vitro [24], and we have previously shown that LUM reduces MMP9 activity in CFB in vitro [21]. However, we found here that the expression of MMP9 and - 2 were not altered in LUM+/- hearts compared to WT, indicating that the profibrotic role of LUM might not be directly through activation of MMPs. GDF15 has been shown to be associated with cardiac fibrosis $[54,55]$ and reported as a diagnostic marker of liver fibrosis [56]. Here, we showed that GDF15 is reduced in LUM+/- post-AB accompanied by reduced fibrosis. Whether GDF-15 might serve as a biomarker in cardiac fibrosis or is mechanistically involved needs further investigation.

Immune cell infiltration and innate immunity play a role in cardiac remodeling and heart failure progression [11, 21, 57-60]. Bacterial lipopolysaccharide LPS and proinflammatory cytokines IL- $1 \beta$ and IL- 6 have been shown to increase LUM expression in CFB in vitro [21]. Moreover, LUM is known to act on macrophages, CFBs, and colonic cells through the NFKB pathway [61, 62]. Here, we demonstrated that a moderate lack of LUM does not affect immune cell infiltration, mRNA expression of inflammatory cytokines IL- 6 and IL- $1 \beta$, and protein levels of NFkB. In line with this, we have recently shown that total LUM deficiency does not affect inflammation, as assessed by immunostaining of mid-ventricular sections with T-lymphocyte and macrophage infiltration markers (CD3 and F4/80, respectively) [24]. Further, we have shown that mRNA expression of immune cell surface and cell adhesion molecules was not affected in the absence or increased level of LUM in vivo and in vitro, respectively.

In summary, a moderate lack of LUM attenuated cardiac fibrosis and improved diastolic dysfunction following pressure overload in mice, adding to the growing body of evidence suggesting that LUM is a central profibrotic molecule in the heart that could serve as a potential therapeutic target.

\section{Acknowledgments}

We are grateful for support and excellent technical assistance from colleagues at the Institute for Experimental Medical Research (IEMR) and Department of Comparative Medicine, Oslo University Hospital.
Mohammadzadeh et al. 


\section{Statement of Ethics}

For the animal experiments, the protocols were reviewed and approved by the Norwegian National Animal Research Committee (protocol IDs 4531, 11669, 7018, and 3170). The NIH Guide for the Care and Use of Laboratory Animals was followed (NIH publication No. 85-23, revised 2011).

\section{Disclosure Statement}

Declarations of interest: none.

\section{Funding Sources}

This work was supported by the Research Council of Norway, Anders Jahre's Fund for the Promotion of Science, the SouthEastern Regional Health Authority, the Norwegian Health asso- ciation, the Kristian Gerhard Jebsen Foundation, the Olav Raagholt and Gerd Meidel Raagholt's Fund for Science, the Rakel and Otto Kristian Bruun's Fund, the Family Blix Fund, the Inger Haldorsens Fund, Norway, the Simon Fougner Hartmanns Family Fund, Denmark, Center for Heart Failure Research, University of Oslo, UNIFOR grants and the LUMKO mouse development was funded by grants from the National Eye Institute (EY11654 to S.C.).

\section{Author Contributions}

Design of the work: N.M., K.V.T.E., T.T., I.G.L., G.C.; data collection: all authors; data analysis and interpretation: all authors; drafting the article: N.M., T.T., I.G.L.; critical revision of the article: all authors; final approval of the version to be published: all authors. N.M. was the project director involved in all aspects and phases of the work.

\section{References}

1 Azevedo PS, Polegato BF, Minicucci MF, Paiva SA, Zornoff LA. Cardiac Remodeling: Concepts, Clinical Impact, Pathophysiological Mechanisms and Pharmacologic Treatment. Arq Bras Cardiol. 2016 Jan;106(1):62-

2 Opie LH, Commerford PJ, Gersh BJ, Pfeffer MA. Controversies in ventricular remodelling. Lancet. 2006 Jan;367(9507):356-67.

3 Dorn GW 2nd. The fuzzy logic of physiological cardiac hypertrophy. Hypertension. 2007 May;49(5):962-70.

4 Hill JA, Olson EN. Cardiac plasticity. N Engl J Med. 2008 Mar;358(13):1370-80.

5 Suthahar N, Meijers WC, Silljé HH, de Boer RA. From Inflammation to Fibrosis-Molecular and Cellular Mechanisms of Myocardial Tissue Remodelling and Perspectives on Differential Treatment Opportunities. Curr Heart Fail Rep. 2017 Aug;14(4):235-50.

6 Frangogiannis NG. Cardiac fibrosis: cell biological mechanisms, molecular pathways and therapeutic opportunities. Mol Aspects Med. 2019 Feb;65:70-99.

7 Schirone L, Forte M, Palmerio S, Yee D, Nocella C, Angelini F, et al. A Review of the Molecular Mechanisms Underlying the Development and Progression of Cardiac Remodeling. Oxid Med Cell Longev. 2017;2017: 3920195.

8 Iozzo RV, Schaefer L. Proteoglycan form and function: A comprehensive nomenclature of proteoglycans. Matrix Biol. 2015;42:11-55.

9 Melleby AO, Strand ME, Romaine A, Herum KM, Skrbic B, Dahl CP, et al. The Heparan Sulfate Proteoglycan Glypican-6 Is Upregulated in the Failing Heart, and Regulates Cardiomyocyte Growth through ERK1/2 Signaling. PLoS One. 2016 Oct;11(10):e0165079.
10 Herum KM, Lunde IG, Skrbic B, Louch WE, Hasic A, Boye S, et al. Syndecan- 4 is a key determinant of collagen cross-linking and passive myocardial stiffness in the pressure-overloaded heart. Cardiovasc Res. 2015 May; 106(2):217-26.

11 Strand ME, Herum KM, Rana ZA, Skrbic B, Askevold ET, Dahl CP, et al. Innate immune signaling induces expression and shedding of the heparan sulfate proteoglycan syndecan-4 in cardiac fibroblasts and myocytes, affecting inflammation in the pressure-overloaded heart. FEBS J. 2013 May;280(10):2228-47.

12 Christensen G, Herum KM, Lunde IG. Sweet, yet underappreciated: Proteoglycans and extracellular matrix remodeling in heart disease. Matrix Biol. 2019 Jan;75-76:286-299.

13 Andenæs K, Lunde IG, Mohammadzadeh N, Dahl CP, Aronsen JM, Strand ME, et al. The extracellular matrix proteoglycan fibromodulin is upregulated in clinical and experimental heart failure and affects cardiac remodeling. PLoS One. 2018 Jul;13(7):e0201422.

14 Kalamajski S, Oldberg A. The role of small leucine-rich proteoglycans in collagen fibrillogenesis. Matrix Biol. 2010;29:248-53.

15 Chakravarti S, Magnuson T, Lass JH, Jepsen KJ, LaMantia C, Carroll H. Lumican regulates collagen fibril assembly: skin fragility and corneal opacity in the absence of lumican. J Cell Biol. 1998 Jun;141(5):1277-86.

16 Pilling D, Vakil V, Cox N, Gomer RH. TNFa-stimulated fibroblasts secrete lumican to promote fibrocyte differentiation. Proc Natl Acad Sci USA. 2015 Sep;112(38):11929-34.

17 Naito Z; Journal of Nippon Medical School =. Role of the small leucine-rich proteoglycan (SLRP) family in pathological lesions and cancer cell growth. J Nippon Med Sch. 2005 Jun;72(3):137-45.
18 Krishnan A, Li X, Kao WY, Viker K, Butters $\mathrm{K}$, Masuoka $\mathrm{H}$, et al. Lumican, an extracellular matrix proteoglycan, is a novel requisite for hepatic fibrosis. Lab Invest. 2012 Dec;92(12): 1712-25.

19 Dupuis LE, Doucette L, Rice AK, Lancaster AE Berger MG, Chakravarti S, et al. Development of myotendinous-like junctions that anchor cardiac valves requires fibromodulin and lumican. Dev Dyn. 2016 Oct;245(10):1029-42.

20 Dupuis LE, Berger MG, Feldman S, Doucette L, Fowlkes V, Chakravarti S, et al. Lumican deficiency results in cardiomyocyte hypertrophy with altered collagen assembly. J Mol Cell Cardiol. 2015 Jul;84:70-80.

21 Engebretsen KV, Lunde IG, Strand ME, Wæhre A, Sjaastad I, Marstein HS, et al. Lumican is increased in experimental and clinical heart failure, and its production by cardiac fibroblasts is induced by mechanical and proinflammatory stimuli. FEBS J. 2013 May; 280(10):2382-98

22 Wæhre A, Halvorsen B, Yndestad A, Husberg C, Sjaastad I, Nygård S, et al. Lack of chemokine signaling through CXCR5 causes increased mortality, ventricular dilatation and deranged matrix during cardiac pressure overload. PLoS One. 2011 Apr;6(4):e18668.

23 Engebretsen KV, Wæhre A, Bjørnstad JL, Skrbic B, Sjaastad I, Behmen D, et al. Decorin, lumican, and their GAG chain-synthesizing enzymes are regulated in myocardial remodeling and reverse remodeling in the mouse. J Appl Physiol (1985). 2013;114:988-97.

24 Mohammadzadeh N, Lunde IG, Andenæs K, Strand ME, Aronsen JM, Skrbic B, et al. The extracellular matrix proteoglycan lumican improves survival and counteracts cardiac dilatation and failure in mice subjected to pressure overload. Sci Rep. 2019 Jun;9(1):9206.
Lumican in Cardiac Remodeling

following Pressure Overload 
25 Jepsen KJ, Wu F, Peragallo JH, Paul J, Roberts L, Ezura Y, et al. A syndrome of joint laxity and impaired tendon integrity in lumicanand fibromodulin-deficient mice. J Biol Chem. 2002 Sep;277(38):35532-40.

26 Melleby AO, Romaine A, Aronsen JM, Veras I, Zhang L, Sjaastad I, et al. A novel method for high precision aortic constriction that allows for generation of specific cardiac phenotypes in mice. Cardiovasc Res. 2018 Oct; 114(12):1680-90.

27 Strand ME, Aronsen JM, Braathen B, Sjaastad I, Kvaløy H, Tønnessen T, et al. Shedding of syndecan-4 promotes immune cell recruitment and mitigates cardiac dysfunction after lipopolysaccharide challenge in mice. J Mol Cell Cardiol. 2015 Nov;88:133-44.

28 Wæhre A, Vistnes M, Sjaastad I, Nygård S, Husberg C, Lunde IG, et al. Chemokines regulate small leucine-rich proteoglycans in the extracellular matrix of the pressure-overloaded right ventricle. J Appl Physiol (1985). 2012; 112:1372-82.

29 Svensson L, Närlid I, Oldberg A. Fibromodulin and lumican bind to the same region on collagen type I fibrils. FEBS Lett. 2000 Mar; 470(2):178-82.

30 Kalamajski S, Oldberg A. Homologous sequence in lumican and fibromodulin leucinerich repeat 5-7 competes for collagen binding. J Biol Chem. 2009 Jan;284(1):534-9.

31 Ezura Y, Chakravarti S, Oldberg A, Chervoneva I, Birk DE. Differential expression of lumican and fibromodulin regulate collagen fibrillogenesis in developing mouse tendons. J Cell Biol. 2000 Nov;151(4):779-88.

32 Yeh JT, Yeh LK, Jung SM, Chang TJ, Wu HH, Shiu TF, et al. Impaired skin wound healing in lumican-null mice. Br J Dermatol. 2010 Dec;163(6):1174-80.

33 Chakravarti S, Petroll WM, Hassell JR, Jester JV, Lass JH, Paul J, et al. Corneal opacity in lumican-null mice: defects in collagen fibril structure and packing in the posterior stroma. Invest Ophthalmol Vis Sci. 2000 Oct;41(11):3365-73.

34 Bjørnstad JL, Sjaastad I, Nygård S, Hasic A, Ahmed MS, Attramadal H, et al. Collagen isoform shift during the early phase of reverse left ventricular remodelling after relief of pressure overload. Eur Heart J. 2011 Jan; 32(2):236-45.

35 López B, González A, Hermida N, Valencia F, de Teresa E, Díez J. Role of lysyl oxidase in myocardial fibrosis: from basic science to clinical aspects. Am J Physiol Heart Circ Physiol. 2010 Jul;299(1):H1-9.

36 Fan D, Takawale A, Lee J, Kassiri Z. Cardiac fibroblasts, fibrosis and extracellular matrix remodeling in heart disease. Fibrogenesis Tissue Repair. 2012 Sep;5(1):15.

37 Dobaczewski M, Bujak M, Zymek P, Ren G, Entman ML, Frangogiannis NG. Extracellular matrix remodeling in canine and mouse myocardial infarcts. Cell Tissue Res. 2006 Jun; 324(3):475-88

38 Heling A, Zimmermann R, Kostin S, Maeno $\mathrm{Y}$, Hein S, Devaux B, et al. Increased expres- sion of cytoskeletal, linkage, and extracellular proteins in failing human myocardium. Circ Res. 2000 Apr;86(8):846-53.

39 van Dijk A, Niessen HW, Ursem W, Twisk JW, Visser FC, van Milligen FJ. Accumulation of fibronectin in the heart after myocardial infarction: a putative stimulator of adhesion and proliferation of adipose-derived stem cells. Cell Tissue Res. 2008 May;332(2):28998.

40 Zhao S, Wu H, Xia W, Chen X, Zhu S, Zhang $S$, et al. Periostin expression is upregulated and associated with myocardial fibrosis in human failing hearts. J Cardiol. 2014 May;63(5): 373-8.

41 Tkatchenko TV, Moreno-Rodriguez RA, Conway SJ, Molkentin JD, Markwald RR, Tkatchenko AV. Lack of periostin leads to suppression of Notch1 signaling and calcific aortic valve disease. Physiol Genomics. 2009 Nov;39(3):160-8.

42 Hernnäs J, Nettelbladt $\mathrm{O}$, Bjermer L, Särnstrand B, Malmström A, Hällgren R. Alveolar accumulation of fibronectin and hyaluronan precedes bleomycin-induced pulmonary fibrosis in the rat. Eur Respir J. 1992 Apr;5(4): 404-10.

43 Muro AF, Moretti FA, Moore BB, Yan M, Atrasz RG, Wilke CA, et al. An essential role for fibronectin extra type III domain A in pulmonary fibrosis. Am J Respir Crit Care Med. 2008 Mar;177(6):638-45.

44 Jarnagin WR, Rockey DC, Koteliansky VE, Wang SS, Bissell DM. Expression of variant fibronectins in wound healing: cellular source and biological activity of the EIIIA segment in rat hepatic fibrogenesis. J Cell Biol. 1994 Dec; 127(6 Pt 2):2037-48.

45 Kaur H, Takefuji M, Ngai CY, Carvalho J, Bayer J, Wietelmann A, et al. Targeted Ablation of Periostin-Expressing Activated Fibroblasts Prevents Adverse Cardiac Remodeling in Mice. Circ Res. 2016 Jun;118(12):1906-17.

46 Valiente-Alandi I, Potter SI, Salvador AM, Schafer AE, Schips T, Carrillo-Salinas F, et al. Inhibiting Fibronectin Attenuates Fibrosis and Improves Cardiac Function in a Model of Heart Failure. Circulation. 2018 Sep;138(12): 1236-52.

47 Conway SJ, Doetschman T, Azhar M. The inter-relationship of periostin, TGF beta, and BMP in heart valve development and valvular heart diseases. ScientificWorldJournal. 2011 Jul;11:1509-24.

48 Lorts A, Schwanekamp JA, Baudino TA, McNally EM, Molkentin JD. Deletion of periostin reduces muscular dystrophy and fibrosis in mice by modulating the transforming growth factor- $\beta$ pathway. Proc Natl Acad Sci USA. 2012 Jul;109(27):10978-83.

49 Huang Y, Liu W, Xiao H, Maitikabili A, Lin $\mathrm{Q}, \mathrm{Wu} \mathrm{T}$, et al. Matricellular protein periostin contributes to hepatic inflammation and fibrosis. Am J Pathol. 2015 Mar;185(3):78697.

50 Kuwahara F, Kai H, Tokuda K, Kai M, Takeshita A, Egashira K, et al. Transforming growth factor-beta function blocking prevents myocardial fibrosis and diastolic dysfunction in pressure-overloaded rats. Circulation. 2002 Jul;106(1):130-5.

51 Ulrich D, Noah EM, von Heimburg D, Pallua N. TIMP-1, MMP-2, MMP-9, and PIIINP as serum markers for skin fibrosis in patients following severe burn trauma. Plast Reconstr Surg. 2003 Apr;111(4):1423-31.

52 Corbel M, Belleguic C, Boichot E, Lagente V. Involvement of gelatinases (MMP-2 and MMP-9) in the development of airway inflammation and pulmonary fibrosis. Cell Biol Toxicol. 2002;18(1):51-61.

53 Van Linthout S, Seeland U, Riad A, Eckhardt O, Hohl M, Dhayat N, et al. Reduced MMP-2 activity contributes to cardiac fibrosis in experimental diabetic cardiomyopathy. Basic Res Cardiol. 2008 Jul;103(4):319-27.

54 Lok SI, Winkens B, Goldschmeding R, van Geffen AJ, Nous FM, van Kuik J, et al. Circulating growth differentiation factor-15 correlates with myocardial fibrosis in patients with non-ischaemic dilated cardiomyopathy and decreases rapidly after left ventricular assist device support. Eur J Heart Fail. 2012 Nov; 14(11):1249-56.

55 Zhou YM, Li MJ, Zhou YL, Ma LL, Yi X. Growth differentiation factor-15 (GDF-15), novel biomarker for assessing atrial fibrosis in patients with atrial fibrillation and rheumatic heart disease. Int J Clin Exp Med. 2015 Nov; 8(11):21201-7.

56 Bellan M, Castello LM, Pirisi M. Candidate Biomarkers of Liver Fibrosis: A Concise, Pathophysiology-oriented Review. J Clin Transl Hepatol. 2018 Sep;6(3):317-25.

57 Frieler RA, Mortensen RM. Immune cell and other noncardiomyocyte regulation of cardiac hypertrophy and remodeling. Circulation. 2015 Mar;131(11):1019-30.

$58 \mathrm{Yu}$ L, Feng Z. The Role of Toll-Like Receptor Signaling in the Progression of Heart Failure. Mediators Inflamm. 2018 Feb;2018:9874109.

59 Frantz S, Falcao-Pires I, Balligand JL, Bauersachs J, Brutsaert D, Ciccarelli M, et al. The innate immune system in chronic cardiomyopathy: a European Society of Cardiology (ESC) scientific statement from the Working Group on Myocardial Function of the ESC. Eur J Heart Fail. 2018 Mar;20(3): 445-59.

60 Frey H, Schroeder N, Manon-Jensen T, Iozzo $\mathrm{RV}$, Schaefer L. Biological interplay between proteoglycans and their innate immune receptors in inflammation. FEBS J. 2013 May; 280(10):2165-79.

61 Wu F, Vij N, Roberts L, Lopez-Briones S, Joyce S, Chakravarti S. A novel role of the lumican core protein in bacterial lipopolysaccharide-induced innate immune response. Biol Chem. 2007 Sep;282(36):26409-17.

62 Lohr K, Sardana H, Lee S, Wu F, Huso DL, Hamad AR, et al. Extracellular matrix protein lumican regulates inflammation in a mouse model of colitis. Inflamm Bowel Dis. 2012 Jan; 18(1):143-51. 